\title{
Die Finanzmarktkrise im Licht einer Theorie funktioneller Differenzierung
}

\author{
Renate Mayntz
}

\begin{abstract}
Zusammenfassung: In diesem Aufsatz geht es um die Frage, ob sich die jüngste Finanzmarktkrise, in Kategorien einer Theorie funktioneller Differenzierung interpretiert, als Erscheinung systemischer Desintegration mit potenziell sozial desintegrativen Folgen verstehen lässt. Dazu wird versucht, ein über die deskriptive Auflistung einzelner Faktoren hinausgehendes, theoretisch fundiertes Verständnis des Prozesses zu gewinnen, der vielfach als Entfesselung oder Verselbständigung des Finanzsystems etikettiert und kritisiert wurde. Theoretisch wird gefragt, ob die „Entfesselung“, die Verselbständigung eines funktionellen Teilsystems, eine spezifische Form von systemischer Desintegration bei funktioneller Differenzierung sein könnte. Praktisch wird gezeigt, dass die sich am Ende krisenhaft manifestierende, von der Politik nicht verhinderte Entwicklung des Finanzsystems das Ergebnis eines Zusammenspiels endogener (Gewinnorientierung der Finanzmarktakteure) und exogener (Nachfrage politischer, ökonomischer und privater Akteure nach Krediten und Anlagemöglichkeiten) Faktoren ist.
\end{abstract}

Schlüsselwörter: Funktionelle Differenzierung · Verselbständigung von Teilsystemen · Akteurinteressen · Finanzsystem · Finanzmarktkrise

\section{The financial crisis in the light of a theory of functional differentiation}

\begin{abstract}
This article tries to answer the question whether the recent financial market crisis, if analyzed in categories of the theory of functional differentiation, can be understood as a process of systemic disintegration with socially disintegrative consequences. Instead of listing descriptively the factors that have been identified as the proximate causes of the financial market crisis, the attempt is made to gain a theoretical understanding of a development that has been interpreted as the de-coupling, the emancipation of the financial system from its service functions. Can this de-coupling, the tendency of actors in the financial system to gain money other than through service to clients be considered a form of systemic disintegration characteristic of functional differentiation? The article shows that the development that led to the recent crisis, a development not prevented by political intervention, is the result of a combination of endogenous and exogenous factors - the profit orientation of financial market actors, and credit seeking by political, economic, and private actors.
\end{abstract}

(C) Springer Fachmedien Wiesbaden 2013

R. Mayntz $(\bowtie)$

Max-Planck-Institut für Gesellschaftsforschung,

Paulstr. 3, 50676 Köln, Deutschland

E-Mail: rm@mpifg.de 
Keywords: Functional differentiation - Uncoupling of functional subsystems - Actor interests · Financial system $\cdot$ Financial market crisis

\section{Die Finanzmarktkrise als Ausdruck mangelhafter Systemintegration?}

In jüngster Zeit wird in Publikationen und Veranstaltungen auffällig oft das Problem des sozialen Zusammenhalts angesprochen. Symptomatisch dafür war der dem Thema Vielfalt und Zusammenhalt gewidmete 36. Kongress der Deutschen Gesellschaft für Soziologie. Im Mittelpunkt stand hier die wachsende Vielfalt von Orientierungsangeboten, sozialen Lagen und Lebensformen, die ebenso wie ethnische und religiöse Diversität den sozialen Zusammenhalt bedroht. Im gleichen Sinn sprechen auch Heitmeyer und Imbusch in einem neuen Sammelband von „Desintegrationsdynamiken“ (Heitmeyer und Imbusch 2012). Hier wie dort werden Desintegrationserscheinungen vorzugsweise auf der Mikroebene festgemacht. Als Gegenkräfte werden vor allem Anerkennung, Vertrauen und soziale Normen angesprochen. Anerkennung spielt in dem Band von Heitmeyer und Imbusch die Rolle eines wichtigen „Integrationsmechanismus“; Vertrauen ist es, was nach dem Titel der WZB-Mitteilungen für März 2012 ,die Gesellschaft und die Welt zusammenhält", und Normen, Standards und Werte sind es, was dem Sammelband von Assmann und anderen zufolge „die Welt zusammenhält“ (Assmann et al. 2012). Offenbar gibt es ein weit verbreitetes Gefühl des Unbehagens und der Verunsicherung: Der Zusammenhang „der Welt", zumindest unserer Welt, erscheint gefährdet.

Aber um welchen Zusammenhang geht es? Im Vordergrund der angeführten Publikationen und des Kongresses der DGS steht - um die bekannte Unterscheidung von David Lockwood (1969) zu benutzen - nicht die Systemintegration, sondern die soziale Integration auf der Mikroebene zwischenmenschlicher Beziehungen. Natürlich besteht, trotz aller Unterschiedlichkeit mikro- und makrosziologischer Perspektiven, eine Verbindung zwischen Desintegrationserscheinungen auf der sozialen Mikroebene und Vorgängen auf der Systemebene. Mängel der sozialen Integration können quasi als Aggregateffekt auch die Systemintegration in Frage stellen. Umgekehrt jedoch könnte fehlende soziale Integration die Folge von Mängeln der Systemintegration sein. Desintegrationserscheinungen auf der gesellschaftlichen Makroebene rücken vor allem dann ins öffentliche Bewusstsein, wenn sie sich krisenhaft auf der Mikroebene auswirken. Ein solcher Fall könnte die Finanzmarktkrise sein, die 2008 manifest wurde und deren direkte und indirekte Folgen Politik und Öffentlichkeit seitdem unablässig beschäftigen.

Politiker und Wissenschaftler haben die Finanzmarktkrise übereinstimmend auf Defizite der Regulierung zurückgeführt. Die Deregulierung der sich internationalisierenden und zugleich strukturell verändernden Finanzindustrie, die seit den 1970er Jahren im Kontext des Neoliberalismus stattfand, hat die Finanzmärkte „entfesselt“, wie es im Titel eines eben erschienenen Buches heißt (Kraemer und Nessel 2012), und so ihre krisenträchtige Dynamik freigesetzt. In der seitdem geführten Debatte um eine Regulierungsreform geht es darum, die „entfesselten“ Finanzmärkte im öffentlichen Interesse wieder unter Kontrolle zu bringen. Die offensichtliche Halbherzigkeit der bislang durchgeführten Reformen wird dem Widerstand der Finanzindustrie, vor allem aber fehlendem politischen Willen zugeschrieben. Aber handelt es sich tatsächlich um einen Mangel 
an Steuerungswillen oder, um eine in den 1970er Jahren geläufige Unterscheidung aufzugreifen, nicht vielmehr um ein Problem der Steuerbarkeit (Mayntz 1987)? Ist das moderne Finanzsystem zumindest in kapitalistischen Demokratien politisch nicht mehr steuerbar? Die öffentliche Debatte deutet in diese Richtung. Die Finanzmärkte, so heißt es im medialen Diskurs, seien außer Kontrolle geraten, das Finanzsystem sei autonom geworden, habe sich verselbständigt. ${ }^{1}$

Außer Kontrolle, autonom, verselbständigt - die Worte scheinen auf Prozesse systemischer Desintegration zu verweisen. Von Soziologen wurde die Finanzmarktkrise aus der Perspektive von Theorien der Finanzialisierung (z. B. Deutschmann 2011) und des modernen Kapitalismus (z. B. Streeck 2011), aber selten in systemtheoretischer Perspektive als Fall versagender Systemintegration gedeutet. Eine Ausnahme bildet das Buch von Langenohl und Wetzel (2012), die versuchen, die Finanzmarktkrise ausdrücklich im Rahmen der Theorie sozialer Differenzierung zu interpretieren. Die Autoren sprechen von der Entkoppelung, dem „Eigenleben“ der Finanzmärkte, die sich vom Rest der Gesellschaft abgelöst hätten. Sie stützen sich bei ihrer Analyse auf Habermas, für den das Integrationsproblem nicht aus der Arbeitsteilung, sondern aus der Differenzierung zwischen System und Lebenswelt folgt. Die Differenzierung zwischen System und Lebenswelt führt nach Habermas in der modernen Gesellschaft zur Herausbildung einer von normativen Zwängen abgelösten Ebene funktionaler Zusammenhänge. Diese verselbständigen sich zu Subsystemen: ,- die rationalisierte Lebenswelt ermöglicht die Entstehung und das Wachstum der Subsysteme, deren verselbständigte Imperative auf sie selbst destruktiv zurückschlagen“ (Habermas 1981, S. 258, 277). Nicht hier setzen jedoch die Autoren an, sondern sie argumentieren, dass der ,verengte Sinn“ des Finanzsystems, nämlich die handlungsleitende Bedeutung von Formalisierungen wie Ratings und die zentrale Rolle von Erwartungen als Motiv von Investitionsentscheidungen, in andere Lebensbereiche „diffundiert“. Wenn hier tatsächlich Diffusion stattgefunden hat, dann dürfte sie allerdings eher in der umgekehrten Richtung, ins Finanzsystem hinein als aus ihm heraus in andere Teilsysteme gelaufen sein. Die wachsende Bedeutung von formalen Kalkülen und die Rolle von Erwartungen bei Unsicherheit sind gesamtgesellschaftliche, mit dem Wissenschaft, Politik und Wirtschaft gleichermaßen betreffenden historischen Rationalisierungsprozess zusammenhängende Tatbestände, die im Finanzsystem eine spezifische, folgenreiche Ausprägung gewonnen haben. Desintegration infolge von Entkoppelung, von Verselbständigung des Finanzsystems ist das jedenfalls nicht. ${ }^{2}$

In der soziologischen Systemtheorie stehen von Spencer über Durkheim zu Parsons, Münch und Luhmann weniger Prozesse der Desintegration als vielmehr die Vorausset-

1 Um nur ein Beispiel zu geben: Andreas Botch, Mitglied des EP und Mitglied von Finance Watch, sprach in der Sendung „Die Welt auf Pump“ am 6. 7. 2011 in 3sat ausdrücklich davon, es müsse verhindert werden, dass die Finanzmärkte sich völlig verselbständigen.

2 Tatsächlich findet sich bei Habermas keine Vorstellung eines verselbständigten Finanzsystems; er betont vielmehr auch in diesem Zusammenhang wieder die Differenzierung zwischen System und Lebenswelt. So heißt es, mit der über das Geldmedium ausdifferenzierten Wirtschaft entstehe ein „sittlich neutralisiertes Handlungssystem“ (Habermas 1981, S. 265), und: „Mit der rechtlichen Institutionalisierung des Geldmediums verliert aber das über egozentrische Nutzenkalküle gesteuerte Erfolgshandeln den Zusammenhang mit verständigungsorientiertem Handeln“(Habermas 1981, S. 292). 
zungen der Systemintegration im Vordergrund. Das folgt nicht zwangsläufig aus der Bezugnahme auf den Begriff des Systems; man könnte anstatt nach den Existenzvoraussetzungen von Systemen auch gezielt nach den Kräften und Mechanismen ihres Zerfalls fragen, so wie Politikwissenschaftler etwa nach dem Zerfall von Imperien fragen. Die zentrale Bedeutung von Integration folgt aus der Interpretation der gesellschaftlichen Evolution als Prozess sozialer Differenzierung, die von segmentärer zu stratifikatorischer und schließlich in der Moderne zu funktioneller Differenzierung, zur Herausbildung funktioneller Teilsysteme führt. ${ }^{3}$ Natürlich unterstellen Systemtheoretiker nicht, dass Integration gelingt, sondern betrachten sie als problematisch. Auch Weber, Simmel und Marx sahen die Möglichkeit, ,dass die Ausdifferenzierung eines gesellschaftlichen Teilsystems zu einem Übermaß an negativen Folgewirkungen in der übrigen Gesellschaft führen kann“" (Schimank 1996, S. 77). Könnte ein solcher Fall die Finanzmarktkrise sein, und ließe sie sich, Luhmann folgend, als Folge selbstreferenzieller, operativer Schließung des Finanzsystems erklären? Im Prozess der Herausbildung verschiedener „Leitdifferenzen“" und spezieller Codes in den (bei Luhmann nicht nur vier) Teilsystemen gewinnen die Teilsysteme „an Autonomie“ (Luhmann 1988, S. 25). Aber Autonomie ist bei Luhmann ein durchaus positiv akzentuierter Begriff; er meint das notwendige Maß an Selbstbestimmung, das funktionelle Differenzierung auf der Ebene von Teilsystemen schon fast logisch voraussetzt (Luhmann 2010, S. 106). So ist auch Selbstreferenz für Luhmann das normale und keineswegs ein pathologisches Ergebnis funktioneller Differenzierung, und solange „strukturelle Kopplung“ funktioniert, bedeutet das keine Desintegration. Das nötige Minimum an Systemintegration wird erreicht, indem störende Umwelteinwirkungen in den Teilsystemen be- und verarbeitet werden. Bei Teubner und Willke (1984) wurde das zur „Kontextsteuerung““.

Im Folgenden geht es um die Frage, ob sich die Finanzmarktkrise, in Kategorien einer Theorie funktioneller Differenzierung interpretiert, als Erscheinung systemischer Desintegration verstehen lässt. Dabei geht es primär um den Versuch, ein über deskriptive Analysen der Finanzmarktkrise hinausgehendes, theoretisch fundiertes Verständnis des als Entfesselung oder Verselbständigung des Finanzsystems etikettierten Prozesses zu gewinnen und nicht um Kritik an einer bestimmten Variante der soziologischen Systemtheorie. ${ }^{4}$ Die Form, die Desintegration auf Systemebene annimmt, hängt mit der Form der je dominanten Differenzierung zusammen: Sezession ist eine Auflösungserscheinung segmentärer Differenzierung, Aufstand bedroht eine stratifizierte Ordnung. Welche Form Desintegration bei funktioneller Differenzierung hat, ist weniger evident; sie wird meist eher implizit als Verfehlen der identifizierten Integrationsvoraussetzungen bestimmt. ${ }^{5}$

3 Habermas (1976, S. 131): „Die funktionalistische Systemtheorie ist der Rahmen, in dem die Soziologie heute an die Evolutionstheorien des 19. Jahrhunderts anknüpft...".

4 Neben den hier angesprochenen Varianten einer Systemtheorie, in der die funktionelle Differenzierung eine zentrale Rolle spielt, gibt es eine an die Kybernetik anschließende Variante (vgl. Greven 1974); von ihr ist hier nicht die Rede.

5 „Den Integrationsbegriff“, so Niklas Luhmann, „,wollen wir negativ definieren als Vermeidung des Umstandes, dass die Operationen eines Teilsystems in einem anderen Teilsystem zu unlösbaren Problemen führen“ (Luhmann 1982, zitiert nach Greve und Kroneberg 2011, S. 8). 
Könnte die „Entfesselung“, die Verselbständigung eines Teilsystems, die spezifische Form von Desintegration bei funktioneller Differenzierung sein?

\section{Finanzmärkte als funktionelles Teilsystem}

Im Unterschied zumindest zu Luhmanns Autonomiebegriff sind Begriffe wie Entfesselung, Entkoppelung und Verselbständigung negativ akzentuiert: Sie sind auf Desintegrationserscheinungen gemünzt. Im ersten Band der Publikationen des Kölner Max-Planck-Instituts, der den Titel „Differenzierung und Verselbständigung“ trägt (Mayntz et al. 1988), wird von der Verselbständigung eines funktionellen Teilsystems gesprochen, wenn es die von Akteuren in seiner Umwelt an es gestellten Leistungserwartungen ,nicht oder nur ungenügend befriedigt" und wenn es zugleich die Fähigkeit zur Abwehr externer Interventionen besitzt, wenn es ,interventionsresistent“" ist (Rosewitz und Schimank 1988, S. 296 f.). ${ }^{6}$ Die nach der Finanzmarktkrise geäußerte Kritik am Finanzsystem entspricht ziemlich genau diesem Konzept von Verselbständigung. Allerdings wird das Finanzsystem in Modellen der funktionell differenzierten Gesellschaft gar nicht als eigenständiges funktionelles Teilsystem behandelt. Von Spencer über Parsons bis zu Luhmann war die Ausdifferenzierung eines besonderen Finanzsystems kein Thema. Natürlich wird gesehen, dass die Wirtschaft intern differenziert ist, so von Parsons und Smelser in „Economy and Society“ (1956), aber auch bei ihnen wird das Finanzsystem nicht gesondert behandelt. Wohl gibt es dort einen „market for capital funds“, aber es geht dabei um Investitionen in das, was heute Realökonomie heißt, in die produzierende Wirtschaft; in Kapitel IV, in dem die ,investment function“ behandelt wird, fehlt jeder Hinweis auf eine mögliche Entkoppelung des Finanzsystems von der produzierenden Wirtschaft. Luhmann betont zwar die Selbstreferentialität des Geldmarktes, der „....weitgehend ohne einen deutlichen Variationszusammenhang mit der äußeren Umwelt"“ operiere (Luhmann 1988). Aber der Code Zahlung/Nichtzahlung, der logisch Geld impliziert, bestimmt für Luhmann das ausdifferenzierte Teilsystem Wirtschaft insgesamt und kann deshalb nicht zugleich die Basis für die Ausdifferenzierung eines besonderen Finanzsystems aus der Wirtschaft sein.

Wenn das Finanzsystem soziologisch nicht als besonderes Teilsystem behandelt wird, dann findet das eine Parallele in der wirtschaftswissenschaftlichen These vom neutralen Geld, der Auffassung, dass Geld ,lediglich als Zahlungs- und Tauschmittel zur Erleichterung der güterwirtschaftlichen Transaktionen fungiert" und es einer speziellen Geldtheorie deshalb nicht bedarf (Kaufmann 1999, S. 228). Den Grund für den blinden Fleck in

6 Diesen Aufsatz hat Uwe Schimank kaum verändert in sein Buch über teilsystemische Autonomie und Gesellschaftssteuerung übernommen (Schimank 2006, S. 119 ff.). Zusätzlich zur ungenügenden Erfüllung von Leistungserwartungen werden 1988 wie 2006 zwei weitere Merkmale von teilsystemischer Verselbständigung genannt, nämlich ein exzessiver Verbrauch von Ressourcen und die Erzeugung gesellschaftlicher Risiken als Nebenfolge der Leistungsproduktion. Dieser weite Begriff wirft nicht nur in dem Aufsatz unbeantwortet bleibende Fragen hinsichtlich der Kausalbeziehung zwischen seinen verschiedenen Komponenten auf; die beiden zusätzlichen Merkmale passen auch nicht recht zur Vorstellung von Entkoppelung, Entfesselung oder Autonomisierung und werden deshalb hier unberücksichtigt gelassen. 
beiden Disziplinen hat Dirk Baecker (1998, S. 108) prägnant formuliert: „Im Unterschied zur Ökonomie denkt die Soziologie die Wirtschaft nicht von Produktion und Konsum, sondern vom Geld her". Während Ökonomen sich auf die Produktion und den Konsum von Waren und Dienstleistungen konzentrieren, wenn sie von Wirtschaft sprechen, und deshalb im Geld nur das Tauschmedium sehen, sehen Soziologen das funktionelle Teilsystem Wirtschaft nicht durch produktive Tätigkeiten, sondern durch das Interesse an Gewinn definiert. Hinter dieser Definition steht das Bemühen, die moderne Wirtschaft, die eine Geldwirtschaft ist, von der Subsistenzwirtschaft in traditionellen Gesellschaften zu unterscheiden. Zugleich wird die moderne Wirtschaft damit implizit als eine kapitalistische verstanden, denn definitionsgemäß ist im Kapitalismus nicht die Produktion von Waren, sondern die Vermehrung des Kapitals, also Gewinn, der letzte Zweck des Handelns. ${ }^{7}$ Wird aber ,Wirtschaft“ differenzierungstheoretisch durch das dominante Interesse an Gewinn bestimmt, dann fehlt eine konzeptuelle Basis für ein eigenständiges Finanzsystem. ${ }^{8}$

Zumindest bei Durkheim und Parsons und anfänglich auch bei Luhmann wurde funktionelle Differenzierung als Arbeitsteilung verstanden (Schimank 1996, S. 156). Hier lässt sich ansetzen, um die Finanzmärkte als ein von der produzierenden Wirtschaft getrenntes funktionelles Teilsystem zu bestimmen. Das Konstitutionskriterium funktioneller Teilsysteme ist, wie jüngst Greve und Kroneberg (2011, S. 9 ff.; vgl. auch Schwinn 2011, S. 427) wieder betont haben, ein immer noch offenes theoretisches Problem, gleichsam die Achillesferse der Theorie sozialer Differenzierung. Der empirisch, und das heißt immer auch historisch denkende Sozialwissenschaftler weiß, dass die Nachfrage nach speziellen Leistungen ebenso wie politisch gesetzte Zwecke (Militär) und kulturelle Sinnangebote (Religion) Anlass zum Entstehen eines spezialisierten Institutionenkomplexes, eines gesellschaftlichen Teilsystems sein können. Wenn man funktionelle Differenzierung als Arbeitsteilung versteht, bieten sich spezielle Leistungen als Kriterium für die Bestimmung funktioneller Teilsysteme an. ${ }^{9}$ Unter diesem Gesichtspunkt betrachtet ist die Güterproduktion das Spezifikum der Wirtschaft. Dem institutionell ausdifferenzierten modernen Finanzsystem werden seinerseits bestimmte Funktionen zugeschrieben; sie werden bestimmt durch die Handhabung von Geld. Geld ist Wertbemessungsstandard, Tauschmittel und Mittel der Wertaufbewahrung oder der zeitlichen Entkoppelung von Produktion und Konsum (Ingham 2004). Das Finanzsystem, so die Funktionszuschreibung, die man auch in der Diskussion um die Finanzmarktkrise immer wieder lesen konnte, organisiert den Zahlungsverkehr und dient der Kapitalallokation: Es bewahrt

7 Die staatssozialistische Planwirtschaft würde nicht unter einen durch Interesse an Gewinn bestimmten Begriff von Wirtschaft passen; das systemtheoretische Konzept funktioneller Differenzierung bezieht sich implizit auf westliche, kapitalistische Gesellschaften.

8 Die Beziehung zwischen Realökonomie und Finanzmärkten steht im Mittelpunkt des Konzepts Finanzmarkt-Kapitalismus, d. h. hier wird zwischen Wirtschaft und Finanzsystem differenziert; von einer (problematischen) „Verselbständigung“ des Finanzsystems ist jedoch nicht die Rede (vgl. Windolf 2005).

$9, \ldots$ die Existenz legitimer Gegenleistungserwartungen und klar umrissener Produzenten- und Abnehmerrollen als Kriterien eines auf Leistungsbasis konstituierten Teilsystems gelten“ (Mayntz 1988, S. 19). 
Erspartes auf und bietet Geldbesitzern die Chance gewinnbringender Investitionen, indem es ihr Geld als Kredit in die produzierende Wirtschaft lenkt (vgl. statt vieler etwa Amato und Fantacci 2012). Diese Leistungen erwarten die Akteure in seiner Umwelt auch tatsächlich von den Institutionen des Finanzsystems. Aber die Finanzinstitutionen hätten sich in ihrem Handeln zunehmend nicht mehr an diesen Leistungserwartungen orientiert, hieß es in Diagnosen der Finanzmarktkrise wie dem viel zitierten LSE Report (Turner et al. 2010).

\section{Verselbständigung als Loslösung von der Erfüllung systemischer Funktionen}

Das Finanzsystem, so könnte man folglich sagen, ,verselbständigt““ sich, wenn sich die Transaktionen von Finanzmarktakteuren von den oben genannten Funktionen lösen, die dem Finanzsystem als Beitrag in einem arbeitsteilig organisierten Sozialsystem zugeschrieben werden. Das geschieht nicht bereits dadurch, dass Banken Geld verleihen, das sie nicht besitzen und so das berühmte „credit money“ schaffen. Es geschieht aber, wenn Banken Gewinn suchen, indem sie, anstatt im Auftrag von Kunden, auf eigene Rechnung miteinander Handel treiben. Mit diesem sogenannten Eigenhandel, der ausschließlich dem Gewinn der Finanzinstitutionen selbst dient und bei dem es vorzugsweise um strukturierte Wertpapiere oder Derivate geht, löst sich das Finanzsystem von den es legitimierenden Leistungen. Nun haben Banken immer schon nicht nur mit Kunden, sondern auch miteinander gehandelt, aber in jüngster Zeit hat eine quantitative Verschiebung in den Transaktionen von Banken zwischen Eigenhandel einerseits und Handel mit und für Kunden andererseits stattgefunden. ${ }^{10}$ Banken haben sich außerdem nicht nur profitorientiert gegenseitig Wertpapiere verkauft, sondern auch gegenseitig Geld geliehen. Im Jahr 2000 haben rund $12 \%$ und 2010 sogar über $23 \%$ der gesamten Transaktionen (Einlagen und Kredite) von regulierten und nicht-regulierten Finanzinstitutionen in der EU innerhalb des Finanzsystems stattgefunden (European Central Bank 2012, S. 21). ${ }^{11}$ Aus der Bankenstatistik der Deutschen Bundesbank geht zugleich hervor, dass im Januar 2012 weit über $50 \%$ der insgesamt erfassten Kredite (3 226814 Mio. €) an (in- und ausländische) Banken gezahlt wurden (1 846353 Mio. €; vgl. Deutsche Bundesbank 2012, S. 20, 26).

Eine Abkehr des Finanzsystems von der Erfüllung der ihm zugeschriebenen Aufgaben findet im Eigenhandel der Banken in reinster Form statt, liegt aber auch schon vor, wenn Banken Transaktionen mit Kunden zur Befriedigung eigener, nicht mit der Aufgabenerfüllung vereinbarer Interessen benutzen. Das geschieht zum Beispiel, wenn Kunden,

10 Diese Verschiebung wird sehr anschaulich beschrieben in Smith (2012, S. 111 ff.). Da in der Bankenstatistik nicht zwischen Handel im Auftrag von Kunden und Handel auf eigene Rechnung unterschieden wird, ist der genaue Umfang des Eigenhandels schwer festzustellen.

11 Man könnte hierin eine Tendenz in Richtung von Modularität oder Zerlegbarkeit sehen; diesem von Herbert Simon (1962) entwickelten Konzept zufolge ist eine Struktur zerlegbar, wenn die Interaktionen zwischen den Subsystemen schwächer sind als die Interaktionen innerhalb der einzelnen Subsysteme. Zerlegbarkeit oder Modularität bedeutet allerdings für Simon nicht Desintegration, sondern erhöht die Stabilität von Systemen. Modularität ist mit funktioneller Differenzierung verwandt, die in der soziologischen Systemtheorie ähnlich wie Arbeitsteilung als leistungssteigernd begriffen wird. 
wie in den USA, zur Aufnahme sogenannter Subprime-Hypotheken gedrängt werden, um Material für strukturierte Wertpapiere für den profitablen Eigenhandel zu gewinnen, oder wenn Banken Wertpapiere verkaufen, die den Käufern absehbar Verlust, gerade dadurch aber den auf diesen Verlust spekulierenden Banken Gewinn bringen. ${ }^{12}$ Derartige Praktiken wurden nach der letzten Krise zum öffentlichen Skandalon und selbst von ausgestiegenen Bankern angeprangert (Smith 2012).

\section{Gewinnorientierung als Triebkraft}

In der öffentlichen Debatte galt die Gier der Banker als Ursache der Finanzmarktkrise. ${ }^{13}$ Könnte es sein, dass finanzielles Gewinnstreben eine für die Akteure im Finanzsystem spezifische, Finanzmarktakteure in besonderem Maße prägende Handlungsorientierung ist?

Die Frage zu stellen setzt voraus, in der Perspektive einer handlungstheoretisch orientierten Differenzierungstheorie zwischen den Eigeninteressen von Akteuren und den an sie als Rollenträger in einem bestimmten funktionellen Teilsystem gerichteten Erwartungen zu unterscheiden. In einem funktionell differenzierten Sozialsystem gibt es in jedem Teilsystem eine doppelte Handlungsorientierung - am Eigeninteresse der individuellen und korporativen Akteure, und an der Erfüllung der dieses Teilsystem definierenden Aufgaben. Das Eigeninteresse individueller und korporativer Akteure kann nicht nur im Finanzsystem zur Abkehr von der Erfüllung der für das Teilsystem spezifischen Aufgaben führen. Eine grundsätzliche Spannung zwischen Eigeninteresse und auf spezifische Leistungen bezogenen normativen Erwartungen ist bei Parsons in der Unterscheidung von self orientation und collectivity orientation angelegt (Parsons 1951, S. 60 f.). Eine handlungstheoretisch fundierte Differenzierungstheorie, wie sie neben anderen bereits Rüschemeyer entwickeln wollte (vgl. Mayntz 1988, S. 11; Schwinn et al. 2011), muss allerdings nicht nur zwischen dem Eigeninteresse von individuellen und korporativen Akteuren einerseits und ihrer Orientierung an den spezifischen Leistungserwartungen an ein Teilsystem andererseits unterscheiden, sondern auch nach soziologisch relevanten Faktoren fragen, die ein Eigeninteresse gerade an finanziellem Gewinn begründen können.

Natürlich wird finanzieller Gewinn oft aus rein persönlichen Gründen gesucht. Das geschieht nicht nur im Finanzsystem; auch in anderen Teilsystemen gewinnt finanzielles Interesse fallweise die Oberhand über die Orientierung an den Aufgaben, etwa wenn Ärzte des Honorars wegen überflüssige Operationen durchführen. Das Streben nach finanziellem Gewinn kann individualpsychologisch erklärt werden, es kann sozialisationsbedingt sein, und es kann kulturell verankert, eine gesellschaftsweit gültige Erwartung sein. Was könnte dazu führen, dass ein anthropologisch verankertes materielles Eigeninteresse sich kulturell als Interesse an finanziellem Gewinn, an „mehr Geld“ manifestiert? In soziolo-

12 In einem solchen Fall („Abacus“) musste Goldman Sachs in den USA schließlich Schadenersatz zahlen; vgl. http://www.tv-replay.de/player/04-09-12/goldman-sachs-eine-bank-lenkt-lenktdie-welt-arte-mediathek60397.html.

13 Zu Gier als Deutungsschema der Krise vgl. Neckel (2011). 
gischen Theorien der Moderne wird verschiedentlich behauptet, die für das Wirtschaftssystem charakteristische ökonomische Handlungsorientierung sei gesellschaftsprägend geworden. ${ }^{14}$ Diese Auffassung findet sich etwa bei Marx, Simmel und Max Weber. ${ }^{15}$ Dass materielles Eigeninteresse sich tatsächlich in immer mehr Bereichen als Interesse an finanziellem Gewinn äußert, ist die unmittelbare Folge der Vermarktlichung, die sich von Bildung und Gesundheit über technische Infrastruktursysteme wie Bahn und Post schließlich auch auf Altenpflege und Freizeitgestaltung ausgedehnt hat. Im öffentlichen Bereich wird die Vermarktlichung durch die Privatisierungspolitik von Regierungen vorangetrieben; im privaten Bereich folgt Vermarktlichung aus der zunehmenden Verfügbarkeit und dem zunehmenden Gebrauch von Geld an Stelle persönlicher Hilfs- und Austauschbeziehungen. Das Streben nach finanziellem Gewinn ist eine Begleiterscheinung von Vermarktlichung, die den Prozess zugleich rekursiv verstärkt. ${ }^{16}$

Dass das Interesse an finanziellem Gewinn im Finanzsystem ein besonderes Gewicht besitzt, hängt auch damit zusammen, dass in jedem Teilsystem die Art der es kennzeichnenden Leistung zur Betonung eines bestimmten Interesses auf der Handlungsebene führt. Im politischen System ist es das Interesse an Macht, denn Macht ist Voraussetzung für die Ausübung einer politischen Funktion. Im Finanzsystem ist, wie in einer Marktwirtschaft generell, finanzieller Gewinn Bestandsvoraussetzung der einzelnen Unternehmen und damit Voraussetzung der Erfüllung von produktiven und von Dienstleistungsaufgaben. Da Finanzinstitutionen außerdem keine materiellen Güter oder fassbare Dienstleistungen wie den Transport von Waren und Personen produzieren, kann finanzieller Gewinn hier besonders leicht zum dominanten Erfolgsmerkmal werden. ${ }^{17}$ Die Diffusion von Gewinnstreben durch Vermarktlichung und die Eigenlogik des Finanzsystems, in die künftige Finanzmarktakteure bereits in der Ausbildung sozialisiert werden, könnten zusammen

14 Luhmann zufolge „,interveniert“ der Geldmarkt in alle anderen Märkte, „,weil überall zur Überbrückung des Zeitabstandes von Ausgaben und Einnahmen Kapital benötigt wird..." (1988, S. 118). Die gesellschaftsweite Diffusion z. B. von Gewinnstreben oder auch von Zweckrationalität ist im Übrigen nicht dasselbe wie die bereichsübergreifende, fundamentalistische Verabsolutierung von einzelnen Wertbezügen (z. B. Heiligkeit, Gesundheit, Umweltschutz), bei der Verantwortungsethik von Gesinnungsethik verdrängt wird; in der Praxis kann die Grenze allerdings fließend sein.

15 Mit Jens Beckert (2009) ließe sich das mit der Tatsache begründen, dass in kapitalistischen Gesellschaften die Dynamik der Wirtschaft eine zentrale Triebkraft der gesellschaftlichen Entwicklung ist.

16 In der Theorie funktioneller Differenzierung geht es zwar eher um die Herausbildung unterschiedlicher Leitwerte als um die gesellschaftsweite Generalisierung einer Handlungsorientierung, doch ließen sich Vermarktlichung oder Finanzialisierung durchaus systemtheoretisch fassen, etwa im Sinne einer pathologischen Form von Interpenetration (vgl. Münch 1991).

17 Neckel (2011) verweist darauf, dass die Besonderheit des nicht mehr auf Kauf oder Verkauf konkreter Objekte bezogene, spekulative Bankgeschäft die Aktivierung eines von der neueren Hirnforschung identifizierten psychischen Mechanismus begünstigt, der Banker dazu bringt, Gewinn gerade durch das Eingehen riskanter Geschäfte zu suchen. 
dazu führen, dass finanzielles Gewinnstreben in diesem System tatsächlich eine besonders starke Motivationskraft besitzt. ${ }^{18}$

\section{Strukturentwicklung als Reaktion auf externe Erwartungen}

Gewinnstreben, wie auch immer bedingt, bleibt als Motiv wirkungslos, wenn es an realen Gewinnchancen mangelt. Die Gewinnchancen im Finanzsystem ergeben sich aus dem Zusammenspiel der Entwicklung von Finanzinstitutionen und den von außen an sie gerichteten Erwartungen. Die heutige Struktur des Finanzsystems hat sich in einem langen historischen Prozess der schrittweisen Ausdifferenzierung entwickelt, zuerst auf der Ebene von Rollen und dann durch das Entstehen von funktionell spezialisierten Institutionen, von Organisationen mit spezifischem Zweckbezug und auf sie bezogenen Regelsystemen. Wie schon in den (von Schimank 1996 behandelten) ,akteurtheoretischen Revisionen“ von Parsons' Differenzierungstheorie durch Rüschemeyer, Eisenstadt oder Smelser betont wurde, ist die konkrete Form, in der die Ausdifferenzierung eines Teilsystems stattfindet, ein vom Eigeninteresse und der relativen Macht einflussreicher externer Akteure beeinflusster historischer Prozess. Ausdifferenzierung, begriffen als historischer Prozess, wendet sich kritisch gegen das die klassische Systemtheorie bestimmende „Dekompositionsparadigma“ funktioneller Differenzierung (Mayntz 1988, S. 14). ${ }^{19}$ Damit fällt dann nicht nur die notwendige Bezugnahme auf Gesellschaft als ein existentes Ganzes, das sich differenziert (vgl. hierzu auch Schwinn 2010, 2011, S. 427), sondern auch die Unterstellung von Funktionalität - und macht damit den Weg frei für die Analyse von unerwünschten, ja desaströsen Entwicklungen (Mayntz 1988, S. 16). So hat auch im Fall des Finanzsystems die Form, die seine Entwicklung speziell seit 1970 genommen hat, am Ende zur jüngsten Finanzmarktkrise geführt.

Diese Krise war wesentlich die Folge einer explodierenden Nachfrage nach Wertpapieren sowie eines ausufernden Kreditgeschäfts, die, begünstigt durch Globalisierung und informationstechnische Innovationen, zu einem explosiven Größenwachstum und einer ständig fortschreitenden internen Differenzierung des Finanzsystems geführt haben. Die Vervielfältigung und wachsende Verbreitung komplexer Wertpapiere mit ihren unterschätzten Risiken wurde stimuliert durch die Zunahme des Anlage suchenden Kapitals, ob es sich um Geld der Golfstaaten oder die Ersparnisse eines wohlhabend gewordenen Mittelstands (Deutschmann 2009) handelt. Nachgefragt wurden Wertpapiere aber auch von der Wirtschaft. Wie Greta Krippner (2011) am Beispiel der USA überzeugend dar-

18 Damit würde nicht nur, Fritz Scharpf folgend, zwischen „public virtues“ oder den Leistungen eines Teilsystems einerseits und den ,private vices“ der in ihm handelnden Akteure andererseits unterschieden, sondern bei den ,private vices“ weiter zwischen einem kulturell akzentuierten und einem von der besonderen Funktionsweise des jeweiligen Teilsystems favorisierten Eigeninteresse (vgl. Scharpf 1988, S. 16, zitiert nach Schimank 2010, S. 233). Gerade diese teilsystemisch favorisierten Eigeninteressen, auch als spezielle Rationalitäten bezeichnet, besitzen offenbar in einem arbeitsteiligen System interdependenter Sektoren eine besondere Sprengkraft.

19 Im Prozess der Ausdifferenzierung entsteht etwas Neues, das zum Teil eines größeren Ganzen erst wird. 
legt, sucht die produzierende Wirtschaft nach Gewinn zunehmend nicht aus dem Verkauf ihrer Produkte, sondern durch Investition auf dem Finanzmarkt; auch hier löst sich, wie beim Eigenhandel der Banken, das Interesse an finanziellem Gewinn von der Erfüllung der Aufgabe - der Aufgabe, Güter und Dienstleistungen zu produzieren.

Parallel zum wachsenden Umfang gehandelter Wertpapiere ist das globale Kreditvolumen gewachsen. Unter den klassischen Finanzfunktionen in der modernen Wirtschaftsgesellschaft stehen die Kreditvergabe und das Investieren in produzierende Unternehmen an erster Stelle. Es sei die ,proper function of finance“, der Realökonomie zu dienen, meinen nicht nur Amato und Fantacci (2012, S. X). Auch Parsons und Smelser bezogen die „,investment function“ auf das als Realökonomie verstandene Wirtschaftssystem; Investmentbanken sammeln ihnen zufolge das Geld von Sparern ein und „commit(s) these funds to firms for investment purposes“" (Parsons und Smelser 1956, S. 218, 233 ff.). Dass die produzierende Wirtschaft in einer an Wachstum orientierten Gesellschaft auf Kredit angewiesen ist, wird allgemein akzeptiert (vgl. z. B. Binswanger 2006). Tatsächlich gehen jedoch Kredite außer an Unternehmen in großem und seit Jahrzehnten wachsendem Maße auch an private Haushalte und an Regierungen. In Deutschland wie in den USA stiegen die an Privatpersonen gezahlten Kredite vor allem seit 1989/90 stark an; in Deutschland machten sie im Jahr 1998 über die Hälfte aller an inländische Unternehmen und Privatpersonen zusammen gezahlten Kredite aus (Bundesbank 2012; vgl. auch Konings 2010, S. 12 ff.; Carruthers und Ariovich 2010, Kap. 4). Ebenso wie Haushalte nehmen auch Regierungen Kredite auf, um laufende Ausgaben zu finanzieren. Fürsten und Könige, die sich Geld vor allem für die Kriegführung borgten, standen am Beginn der Geldentwicklung, und es war königlicher Geldhunger, der bei der Geburt der Bank of England, der ersten Zentralbank, Pate stand (Graeber 2011; Jongschul 2011). Seit Mitte der 1970er Jahre ist die Staatsverschuldung im Durchschnitt der zwölf wichtigsten OECD-Länder kontinuierlich gestiegen - von etwa $40 \%$ des Sozialprodukts zu Beginn der Periode bis auf über $90 \%$ im Jahr 2010 (Streeck 2013).

Die wachsende Nachfrage nach Krediten einerseits und nach Anlagemöglichkeiten andererseits bot Finanzinstitutionen Gewinnchancen, die sie ohne solche Nachfrage nicht gehabt hätten, und regte ihre Vervielfältigung und ihr Größenwachstum an. Spezialisierte Kreditanstalten hat es vor allem im Agrarsektor und bei der Baufinanzierung schon lange gegeben, aber nach dem Zweiten Weltkrieg wuchs ihre Vielfältigkeit: Es entstanden Geldmarktfonds und Beteiligungsgesellschaften, die berüchtigten „Heuschrecken“, und der Anteil des Investmentbanking am Bankgeschäft stieg. 1950 gab es in Deutschland nur 2 Investmentfonds; fünzig Jahre später zählte man 1876 sogenannte Publikumsfonds und weitere 5764 Spezialfonds (http://www.bvi.de/statistikwelt/investmentstatistik). Das Eigeninteresse der Akteure in der Umwelt des Finanzsystems, ihr Interesse an günstigen Krediten, an profitabler Geldanlage und an Risikobegrenzung hat bestimmt, woran sich finanzielles Gewinnstreben im Finanzsystem festmachen konnte. Zynisch könnte man sagen, dass jede Gesellschaft nicht nur die Regierung, sondern auch das Finanzsystem hat, das sie verdient.

Die Ausdifferenzierung eines Teilsystems ist keine Reaktion auf theoretisch abgeleitete, systemische Funktionserfordernisse, sondern auf eine reale externe Nachfrage. Die Entwicklung, die das ausdifferenzierte Finanzsystem genommen hat, wurde systemgefährdend - aber nicht einfach, weil die Finanzinstitutionen sich dem Eigenhandel 
zugewandt und nicht mehr primär an ihren Dienstleistungsfunktionen orientiert hätten, sondern weil sie dem gewachsenen Kundeninteresse an Krediten, Anlagemöglichkeiten und Risikostreuung entsprochen haben, das ihnen neue Gewinnchancen bot. Die in jedes funktionell differenzierte Sozialsystem eingebaute Disziplinierung von Tendenzen zur Abkoppelung der Teilsysteme von ihren spezifischen Leistungen hat diese Entwicklung nicht verhindern können. ${ }^{20}$ Dasselbe gilt für die sanktionsbewehrten Normen, die, auf Gesetze gestützt oder in Form einer professionellen Ethik, in fast allen Teilsystemen der Ablösung von ihren spezifischen Leistungen entgegenwirken sollen. ${ }^{21}$ Im Finanzsystem wird finanzielles Gewinnstreben etwa in Form des Insiderhandels durch Normen der Marktintegrität diszipliniert. Aber im Zuge der der Finanzindustrie in der Ära neoliberaler Politik konzedierten Selbstregelung haben sich krisenträchtige Strukturen, Finanzinstrumente und Praktiken entwickelt und verbreitet. Die komplexen strukturierten Wertpapiere, Hedgefonds und Zweckgesellschaften, die in diesem Freiraum entstanden, blieben unreguliert. Wie schon in früheren Banken- und Finanzkrisen führte deshalb auch die jüngste Krise zum Ruf nach politischer Intervention ins Finanzsystem. Die seitdem eingeleiteten Reformen der Finanzmarktregulierung haben jedoch lediglich zu inkrementellen Veränderungen geführt, die neue Krisen nach ähnlichem Muster kaum verhindern werden (Mayntz 2012). Haben wir es hier schlicht mit Politikversagen zu tun oder ist das Finanzsystem ,interventionsresistent"?

\section{Steuerungsdefizit oder Interventionsresistenz?}

Systemtheoretisch betrachtet, ist es die Aufgabe des bei Parsons am Kriterium des goalattainment orientierten politisch-administrativen Teilsystems, die Leistungserbringung (performance) anderer Teilsysteme $\mathrm{zu}$ sichern und $\mathrm{zu}$ verhindern, dass ihr Operieren negative Externalitäten erzeugt. Ein Politikversagen ließe sich dann als mangelhafte Funktionserfüllung des politischen Teilsystems beschreiben. Niklas Luhmann bestritt bekanntlich grundsätzlich die gesellschaftliche Steuerungsfähigkeit des politischen Teilsystems. ${ }^{22}$ Für jüngere Systemtheoretiker ist politische Steuerung ein wichtiges Thema, aber auch ihnen geht es vor allem um ihre Einschränkungen. ${ }^{23}$ In der akteurtheoretisch

$20 \mathrm{Im}$ Finanzsystem wie in der produzierenden Wirtschaft findet finanzielles Gewinnstreben eine Grenze in der Tatsache, dass die Befriedigung einer wie auch immer zustande gekommenen Nachfrage nach konkreten Dienstleistungen oder Gütern Voraussetzung für das Erzielen von Gewinn ist. In der Politik hat die Abhängigkeit des erstrebten Machterwerbs oder Machterhalts von Wahlen diese disziplinierende Wirkung. Wenn die Befriedigung des Eigeninteresses der Teilsystemakteure an die Erfüllung der das Teilsystem definierenden Leistung gekoppelt ist, wirkt sozusagen eine umgekehrte ,visible hand“.

21 Auf Luhmann gestützt verweist auch Schimank (2006, S. 124 ff.) auf Grenzen der Verselbständigung, die aus der funktionellen Differenzierung resultieren; dazu gehört Ressourcenknappheit und „Reflexion“, aus der Selbstdisziplinierung erwachsen kann.

22 Siehe hierzu das Streitgespräch zwischen Niklas Luhmann und Fritz W. Scharpf in Hartwich (1989, S. 12 ff.).

23 Von Helmut Willkes drei Bänden zur Systemtheorie (Fischer UTB 1993-1996) sind zwei der Intervention in oder Steuerung von komplexen (vor allem sozialen) Systemen gewidmet. 
argumentierenden Theorie politischer Steuerung werden Gründe für Defizite bei der Lösung systemgefährdender Probleme sowohl aufseiten der einer Intervention widerstehenden Steuerungsobjekte wie aufseiten der Steuerungssubjekte, ihrem mangelnden Wollen und Können gesehen.

Interventionsresistenz am Widerstand von Steuerungsadressaten, im konkreten Fall der Finanzindustrie, festzumachen, bringt zwangsläufig Macht ins Spiel. Der Systemtheorie wird gern Machtblindheit vorgeworfen; tatsächlich vernachlässigt sie eher Akteure. Macht ist bei Parsons Steuerungsmedium, bei Luhmann Kommunikationsmedium, aber erst, wenn man anstatt von Funktionen oder Leitwerten von Akteuren ausgeht und funktionelle Differenzierung konkret als Arbeitsteilung begreift, wird Macht zu einer zentralen Dimension. Arbeitsteilung kann Kooperation und gegenseitige Ergänzung bedeuten, impliziert aber immer Abhängigkeit und Ungleichheit. Funktionelle Differenzierung nach dem Teammodell, bei dem alle Mitglieder gleichwertig sind und niemand befiehlt, ist in der Realität gesellschaftlicher Arbeitsteilung ein Grenzfall. Funktionelle Differenzierung und ungleiche Verteilung von Macht sind eng miteinander verschränkt, wie schon Crozier und Friedberg (1979) konkret am Beispiel formaler Organisationen gezeigt haben. Wer bei der Betrachtung der modernen Gesellschaft nur die funktionelle Differenzierung sieht und ihr Zusammenwirken mit stratifikatorischer Differenzierung, der Dimension gesellschaftlicher Ungleichheit vernachlässigt, ist dafür blind.

In der Binnenstruktur des Finanzsystems bilden die CEOs von Finanzinstitutionen eine in sich noch einmal geschichtete Funktionselite. Ihre Entscheidungen setzen die Rahmenbedingungen für das Handeln anderer Akteursgruppen, sowohl innerhalb wie außerhalb des Finanzsystems. Für Konings (2010) sind es deshalb auch nicht die Finanzmärkte, sondern es ist die finanzielle Elite, die Macht ausübt, indem sie die Märkte steuert. Eine allein auf unmittelbare Machtbeziehungen, die Macht von Finanzinstitutionen wie Goldman Sachs oder der von Staranwälten unterstützten Lobby der Finanzindustrie abstellende Analyse greift jedoch zu kurz. Interventionsresistenz, das zweite der oben genannten Definitionsmerkmale von Verselbständigung, wird spürbar erst auf dem Hintergrund gewollter Intervention. Aber wie der Volksmund weiß: Auch wollen muss man können. Der entscheidende Grund für die noch immer unzureichende Regulierung des Finanzsystems liegt in der inzwischen öffentlich sichtbar gewordenen Abhängigkeit der Politik vom Finanzsystem. Je abhängiger externe Akteure von den Operationen einer bestimmten Institution sind, umso leichter kann diese sich gegen ihre Interventionen wehren. Das Finanzsystem ist politisch nicht mehr steuerbar, ist ,interventionsresistent“", weil die Politik von ihm abhängig geworden ist. Da nationale politische Instanzen trotz der Internationalisierung des Finanzsystems nach wie vor die entscheidenden Regulierer sind (vgl. Mayntz 2010), manifestiert sich diese Abhängigkeit in doppelter Hinsicht auf nationaler Ebene. Hier hängt die Politik zum einen von den Steuern ab, die die Finanzindustrie abwirft, von den Jobs und den Wettbewerbsvorteilen vor anderen Ländern, die sie schafft, und vom ökonomischen Wachstum das sie fördert. Alle verfügbaren Indikatoren zeigen eine wachsende gesamtwirtschaftliche Bedeutung des Finanzsektors, egal ob man die Zahl der Beschäftigten, seinen Anteil am Einkommen, am Bruttosozialprodukt oder am Steueraufkommen nimmt. Zum anderen hängt die Politik, hängen Regierungen vom Finanzsystem als Schuldner ab. Als die Fürsten Geld brauchten, um Söldner zu bezahlen, brachte Machtinteresse sie in finanzielle Abhängigkeit; heute ist es in demokra- 
tischen Systemen immer noch Machtinteresse, das die Politik veranlasst, zwecks Erfüllung eigentlich unbezahlbarer Wünsche des Wahlvolks, Geld aufzunehmen. Nach einem kurzen Moment, in dem es manchen Beobachtern schien, der Staat habe beim Krisenmanagement der Jahre 2008 und 2009 die ihm schon abgesprochene Handlungsfähigkeit bewiesen, machte die mit der Finanzmarktkrise verknüpfte Staatsschuldenkrise das ganze Ausmaß seiner Abhängigkeit vom Finanzsystem sichtbar. Seitdem ist weniger von einer Abkoppelung des Finanzsystems als davon die Rede, es diktiere der Politik, was sie zu tun habe. ${ }^{24}$ Eine über Geld vermittelte Abhängigkeit der Politik vom Finanzsystem hat in einer Theorie, die Geld nur als Tauschmedium behandelt und nicht zwischen Wirtschaft und Finanzsystem unterscheidet, keinen rechten Platz.

Neben der Abhängigkeit der Politik vom Finanzsystem steht mangelndes Wissen einer regulierenden Intervention im Wege: Die „Interventionsresistenz“ des modernen Finanzsystems ist auch das Ergebnis seiner Komplexität und der damit verbundenen Intransparenz (vgl. u. a. Willke 2011). Die Bewegungen von Aktienkursen, Risikoaufschlägen für Anleihen und dem Buchwert strukturierter Wertpapiere sind das emergente Resultat komplex verbundener Entscheidungen einer Vielzahl heterogener Akteure. Offensichtlich versteht nicht einmal die zuständige Disziplin die komplexen Interaktionen in diesem System. Rhetorisch werden Finanzmärkte zwar - vor allem im Kontext der europäischen Staatschuldenkrise - als Akteur ${ }^{25}$ behandelt, der belohnt oder straft, aber die öffentlichen Begründungen für konkrete politische Entscheidungen sprechen, wenn man genau hinhört, eine andere Sprache: Hier erscheinen Finanzmärkte als unpersönliches, abstraktes Kollektiv, auf das man lediglich durch die Manipulation von Rahmenbedingungen, durch das Setzen von Anreizen einwirken kann, es ist ein System das einem gegenübertritt wie die Natur. Diese konzeptionelle „Naturalisierung“ des Finanzsystems verschleiert handfeste Machtbeziehungen und dient zugleich als Entschuldigung für selbstverschuldete politische Ohnmacht.

\section{Differenzierungstheorie und Finanzmarktkrise: Eine Coda}

Um zur Ausgangsfrage zurückzukommen: Lässt sich die Finanzmarktkrise mit ihren ökonomischen und den hier nicht weiter behandelten, aber unzweifelhaft damit verbundenen sozial desintegrativen Folgen als Desintegrationserscheinung auf Systemebene deuten, und bietet eine Theorie funktioneller Differenzierung einen analytischen Rahmen für die Erklärung der zu der Krise führenden Entwicklung des Finanzsystems? Eine vollständige Beantwortung dieser Frage würde eine theoretische Beschäftigung mit den verschiedenen Formen und Faktoren von Desintegration in funktionell differenzierten Systemen vor-

24 So stand das Max-Planck Forum am 5. 11. 2012 In Berlin unter dem Thema „Regiert der Markt den Staat?“ Vgl. auch die Tagung „Die Macht des Geldes und die Ohnmacht der Politik“ des Instituts für Zukunftsstudien und Technologiebewertung am 20. 9. 2011 in Berlin.

25 Hier handelt es sich um eine andere Art von „Akteurfiktion“ als die von Schimank herausgestellte Prägung individuellen Handelns im Kontext eines gesellschaftlichen Teilsystems durch die für es spezifischen evaluativen, normativen und kognitiven Orientierungen (Schimank 1988). 
aussetzen, die den selbst gesteckten Rahmen dieses Aufsatzes weit überschritten hätte. Stattdessen wurde davon ausgegangen, dass es sich bei der Finanzmarktkrise von 2008 tatsächlich um eine Fehlentwicklung des Finanzsystems mit potenziell systemgefährdenden Folgen gehandelt hat, eine politisch und im öffentlichen Krisendiskurs unbestrittene Deutung des Geschehens.

Mangelhafte Systemintegration wurde begrifflich zunächst festgemacht am Konzept der Verselbständigung, der Loslösung eines Teilsystems innerhalb eines funktionell differenzierten Ganzen von seinen für andere Teilsysteme und damit für den Erhalt dieses Ganzen erforderlichen Leistungen. Dieser Ansatz ist schon deshalb unzureichend, weil die so verstandene Verselbständigung eines Teilsystems nur eine theoretisch mögliche Form von Desintegration bei funktioneller Differenzierung darstellt. Andere Formen wären eine Dominanz des politischen Teilsystems, wie sie in totalitären Regimen zur Entdifferenzierung führt, oder die von Uwe Schimank (2006, S. 71 ff.) behandelte „feindliche Übernahme“" eines Teilsystems durch ein anderes. Davon abgesehen ist das Konzept von Verselbständigung selbst problematisch. Verselbständigung ist eine Variable, kein Zustand. In einem System, dessen Bestandteile von vornherein locker und zugleich prekär gekoppelt sind, ist die „Verselbständigung“ eines Teils eine immer vorhandene Tendenz. Sie wird erst dann als Desintegrationserscheinung wahrgenommen, wenn sie schwerwiegende negative Auswirkungen auf andere Teilsysteme oder auf den Zusammenhalt des Ganzen hat. Inhaltlich problematisch ist, dass der in Abschn. 2 und Abschn. 3 beschriebene Prozess der Verselbständigung stillschweigend von einem Verständnis funktioneller Differenzierung als einer vom Streben nach Effizienz angetriebenen Arbeitsteilung ausgeht und Verselbständigung als einen endogen, durch das Gewinnstreben der Finanzmarktakteure angetriebenen Prozess betrachtet, der den Leistungsaustausch in einem solchen System untergräbt. Das hier aufscheinende, auf der klassischen Systemtheorie basierende Konzept funktioneller Differenzierung kann, auf abgegrenzte Institutionenkomplexe bezogen, sehr wohl als normativer Standard dienen, an dem man das Ergebnis historischer Entwicklungsprozesse messen kann; es kann jedoch konkrete historische Ereignisse wie die Finanzmarktkrise nicht kausalgenetisch erklären.

Anders verhält es sich mit handlungstheoretischen Konzepten funktioneller Differenzierung. Ein auf den Fall der Finanzmarktkrise bezogenes erklärungsstarkes Konzept funktioneller Differenzierung geht von Leistung im Sinne konkret nachgefragter oder doch tolerierter, spezifischer Aktivitäten als Differenzierungskriterium aus. Es betrachtet das Finanzsystem auf dieser Basis als ein von der produzierenden Wirtschaft getrenntes System und bezieht nicht nur die Eigeninteressen der Akteure im Teilsystem, sondern gleichermaßen die von externen Akteuren tatsächlich nachgefragten Leistungen in die Analyse ein. Es ist immer die konkrete Nachfrage, die unter bestimmten z. B. rechtlichen und technischen Zusatzbedingungen die Form bestimmt, in der sich ein auf spezifischen Leistungen basierendes Teilsystem ausdifferenziert und weiter entwickelt. Die Befriedigung einer externen Nachfrage bietet zugleich Chancen für die Befriedigung individueller und korporativer Eigeninteressen der „Anbieter“. Die so, im Zusammenspiel von internen und externen, gleichermaßen eigeninteressierten Akteuren entstandene Struktur und Operationsweise des (internationalisierten) Finanzsystems wurde systembedrohend. Aber die Finanzkrise wurde nicht durch die Entkoppelung von Gewinnstreben und Aufgabenerfüllung, durch die Instrumentalisierung von Dienstleistungsaufgaben im 
finanziellen Eigeninteresse der Finanzakteure hervorgerufen, sondern durch den Aufbau von Institutionen und die Entwicklung von Finanzinstrumenten zur gewinnorientierten Nutzung einer bestimmten externen Nachfrage. Hier findet, genau betrachtet, eben gerade keine ,Verselbständigung“ im Sinne der Herauslösung eines Teilsystems aus dem Interaktionszusammenhang mit seiner sozialen Umwelt statt.

Auch von Interventionsresistenz des Finanzsystems zu sprechen, ist problematisch. Interventionsresistenz gilt zwar als Merkmal des „,verselbständigten“ Teilsystems, aber im Fall des Finanzsystems wird dieses Merkmal nicht allein durch seine komplexe Struktur und seine schwer durchschaubare Operationsweise bestimmt, sondern durch Faktoren aufseiten des politischen Systems mitbedingt. Es ist nicht so, dass die Politik die Entwicklungen, die am Ende zur Finanzmarktkrise von 2008 führten, nicht hätte unterbinden können, wenn sie es denn gewollt hätte. Aber das politische Wollen wurde durch mangelndes Wissen und die mehrfache Abhängigkeit von Leistungen des Finanzsystems gelähmt. Wollen können ist eine entscheidende Voraussetzung politischen Handelns. Wenn Regierungen vom Finanzsystem abhängiger sind als dieses von ihnen, beeinträchtigt das ihre Fähigkeit zur problemlösenden Intervention. Die Interventionsresistenz des Finanzsystems ist deshalb nicht als quasi objektives Merkmal des Finanzsystems zu verstehen: Sie ist ein relationales, auf die Beziehung zwischen Politik und Finanzsystem bezogenes Merkmal. Diese Relation ist am Ende ausschlaggebend für die mit dem Begriff der Verselbständigung verbundene Gefahr von Desintegration.

Die Entwicklung, die das internationalisierte Finanzsystem in den letzten Jahrzehnten genommen hat, führt nicht zu Desintegration im Sinne des Zerfalls eines Ganzen in seine Teile - so sieht Desintegration nur bei segmentärer Differenzierung aus. Politisches System, Finanzsystem und Realökonomie haben sich nicht voneinander entkoppelt, sondern sie sind kausal betrachtet aufs Innigste miteinander verflochten, ja verklammert. Nicht Entkoppelung, sondern komplexe Interdependenz zwischen im Luhmann'schen Sinn autonomen Teilsystemen ist das Problem. Nicht zufällig fällt in der Debatte um die Ursachen der Finanzkrise und die Schwierigkeiten der politischen Reform des Finanzsystems immer wieder der Hinweis auf seine Komplexität. Dem könnte ein analytischer Ansatz gerecht werden, der die komplexen Zusammenhänge zwischen Finanzsystem, Wirtschaft und Politik in den Mittelpunkt stellt und ausdrücklich nach Formen und Ursachen von Desintegration fragt. Desintegration auf sozialer wie auf Systemebene findet allerdings nicht nur bei funktioneller Differenzierung statt; die ethnisch, religiös und politisch motivierten Konflikte, die im Augenblick vor allem im Nahen Osten und in Afrika auftreten, haben mehr mit Desintegrationserscheinungen bei segmentärer und stratifikatorischer als bei funktioneller Differenzierung zu tun. Wenn Erklärung realer historischer Ereignisse das Ziel ist, genügt kein Ansatz, der allein auf funktionelle Differenzierung abstellt; vielmehr müsste die Verschränkung der verschiedenen Differenzierungsformen in die Erklärung einbezogen werden.

Danksagung: Ich bedanke mich bei Fritz W. Scharpf, Uwe Schimank und den anonymen Gutachtern für hilfreiche kritische Hinweise. 


\section{Literatur}

Amato, Massimo, und Luca Fantacci. 2012. The end of finance. Cambridge. UK: Polity Press.

Assmann, Heinz-Dieter, Frank Baasner und Jürgen Wertheimer. Hrsg. 2012. Normen, Standards, Werte - Was die Welt zusammenhält. Baden-Baden: Nomos.

Baecker, Dirk. 1998. Die Unruhe des Geldes, der Einbruch der Frist. In Rätsel Geld. Annäherungen aus ökonomischer, soziologischer und historischer Sicht, Hrsg. Waltraud Schelkle und Manfred Nitsch, 107-124. Marburg: Metropolis.

Beckert, Jens. 2009. Wirtschaftssoziologie als Gesellschaftstheorie. Zeitschrift für Soziologie 38:182-197.

Binswanger, H. C. 2006. Die Wachstumsspirale: Geld, Energie und Imagination in der Dynamik des Marktprozesses. Marburg: Metropolis-Verlag.

Bundesbank. 2012. Statistiken, Zeitreihen-Datenbanken, Zeitreihe BBK01.PQA355, Kredite an inländische Unternehmen und Privatpersonen; Kredite an inländische Unternehmen/insgesamt/alle Bankengruppen.

Carruthers, Bruce G., und Laura Ariovich. 2010. Money and credit. A sociological approach. Cambridge. UK: Polity Press.

Crozier, Michel, und Erhard Friedberg. 1979. Macht und Organisation. Königstein i. Taunus: Athenäum.

Deutschmann, Christoph. 2009. Soziologie kapitalistischer Dynamik. Köln: MPIfG Working Paper $09 / 5$.

Deutschmann, Christoph. 2011. Limits to financialization. Sociological analyses of the financial crisis. European Journal of Sociology 52:347-389.

European Central Bank. 2012. Shadow banking in the Euro area - An overview. Occasional Paper Series No. 133/April 2012.

Graeber, David. 2011. Debt: The first 5000 years. New York: Melville House.

Greve, Jens, und Clemens Kroneberg. 2011. Herausforderungen einer handlungstheoretisch fundierten Differenzierungstheorie - zur Einleitung. In Soziale Differenzierung. Handlungstheoretische Zugänge in der Diskussion, Hrsg. Clemens Kroneberg, Thomas Schwinn und Jens Greve, 7-23. Wiesbaden: VS Verlag für Sozialwissenschaften.

Greven, Michael Th. 1974. Systemtheorie und Gesellschaftsanalyse. Darmstadt: Luchterhand.

Habermas, Jürgen. 1981. Theorie des kommunikativen Handelns, Bd. 2 - Zur Kritik der funktionalistischen Vernunft. Frankfurt a. M.: Suhrkamp.

Habermas, Jürgen. 1976. Zur Rekonstruktion des Historischen Materialismus. Frankfurt a. M.: Suhrkamp.

Hartwich, Hans-Hermann. Hrsg. 1989. Macht und Ohnmacht politischer Institutionen. 17. Wissenschaftlicher Kongress der DVPW. Wiesbaden: Westdeutscher Verlag.

Heitmeyer, Wilhelm, und Peter Imbusch. Hrsg. 2012. Desintegrationsdynamiken. Integrationsmechanismen auf dem Prüfstand. Wiesbaden: Springer VS.

Ingham, Geoffrey. 2004. The nature of money. Cambridge: Polity.

Jongschul, Kim. 2011. How modern banking originated: The London Goldsmith-bankers' institutionalisation of trust. Business History 53:939-959.

Kaufmann, Franz-Xaver. 1999. Kritik des neutralen Geldes. Geschichte und Gesellschaft 25:226-251.

Konings, Martin. 2010. Rethinking neoliberalism and the crisis. Beyond the re-regulation agenda. In The great credit crash, Hrsg. Martin Konings, 1-30. London: Vero.

Kraemer, Klaus, und Sebastian Nessel. Hrsg. 2012. Entfesselte Finanzmärkte. Soziologische Analysen des modernen Kapitalismus. Frankfurt a. M.: Campus.

Krippner, Greta R. 2011. Capitalizing on crisis. The political origins of the rise of finance. Harvard: University Press. 
Langenohl, Andreas, und Dietmar J. Wetzel. 2012. Die Entgrenzung von Nicht-Sinn: Zur Konzipierung entfesselter Finanzmärkte. In Entfesselte Finanzmärkte. Soziologische Analysen des modernen Kapitalismus, Hrsg. Klaus Kraemer und Sebastian Nessel, 63-81. Frankfurt a. M.: Campus.

Lockwood, David. 1969. Soziale Integration und Systemintegration. In Theorien des sozialen Wandels, Hrsg. Wolfgang Zapf, 124-137. Köln: Kiepenheuer.

Luhmann, Niklas. 1988. Die Wirtschaft der Gesellschaft. Frankfurt a. M.: Suhrkamp.

Luhmann, Niklas. 2010. Politische Soziologie. Frankfurt a. M.: Suhrkamp.

Mayntz, Renate. 1987. Politische Steuerung und gesellschaftliche Steuerungsprobleme. Anmerkungen zu einem theoretischen Paradigma. In Jahrbuch zur Staats- und Verwaltungswissenschaft Bd. 1, 89-110. Baden-Baden: Nomos.

Mayntz, Renate. 1988. Funktionelle Teilsysteme in der Theorie sozialer Differenzierung. In Differenzierung und Verselbständigung. Zur Entwicklung gesellschaftlicher Teilsysteme, Hrsg. Renate Mayntz et al., 11-44. Frankfurt a. M.: Campus.

Mayntz, Renate. 2010. Die Handlungsfähigkeit des Nationalstaats bei der Regulierung der Finanzmärkte. Leviathan 38:175-187.

Mayntz, Renate. 2012. Crisis and control. Institutional change in financial market regulation. In Crisis and control. Institutional change in financial market regulation, Hrsg. Renate Mayntz, 29-65. Frankfurt a. M.: Campus.

Mayntz, Renate, Bernd Rosewitz, Uwe Schimank und Rudolf Stichweh. 1988. Differenzierung und Verselbständigung. Zur Entwicklung gesellschaftlicher Teilsysteme. Frankfurt a. M.: Campus.

Münch, Richard. 1991. Dialektik der Kommunikationsgesellschaft. Frankfurt a. M.: Suhrkamp.

Neckel, Sighard. 2011. Der Gefühlskapitalismus der Banken: Vom Ende der Gier als „,ruhiger Leidenschaft". Leviathan 39:39-53.

Parsons, Talcott. 1951. The social system. Glencoe: Free.

Parsons, Talcott, und Neil J. Smelser. 1956. Economy and society: A study in the integration of economic and social theory. London: Routledge.

Rosewitz, Bernd, und Uwe Schimank. 1988. Verselbständigung und politische Steuerbarkeit gesellschaftlicher Teilsysteme. In Differenzierung und Verselbständigung. Zur Entwicklung gesellschaftlicher Teilsysteme, Hrsg. Renate Mayntz et al., 295-329. Frankfurt a. M.: Campus.

Scharpf, Fritz W. 1988. Verhandlungssysteme, Verteilungskonflikte und Pathologien der politischen Steuerung. Köln: MPIfG Working Paper 88/1.

Schimank, Uwe. 1988. Gesellschaftliche Teilsysteme als Akteurfiktionen. Kölner Zeitschrift für Soziologie und Sozialpsychologie 40:619-639.

Schimank, Uwe. 1996. Theorien gesellschaftlicher Differenzierung. Opladen: Leske \& Budrich.

Schimank, Uwe. 2006. Teilsystemische Autonomie und politische Gesellschaftssteuerung. Beiträge zur akteurzentrierten Differenzierungstheorie 2. Wiesbaden: VS Verlag für Sozialwissenschaften.

Schimank, Uwe. 2010. Reputation statt Wahrheit: Verdrängt der Nebencode den Code? Soziale Systeme 16:233-242.

Schwinn, Thomas. 2010. Wirtschaftssoziologie als Gesellschaftstheorie? Kritische Anfragen aus einer Weber'schen Perspektive. In Wirtschaftssoziologie nach Weber, Hrsg. Andrea Maurer, 199-225. Wiesbaden: VS Verlag für Sozialwissenschaften.

Schwinn, Thomas. 2011. Perspektiven der neueren Differenzierungstheorie. In Soziale Differenzierung. Handlungstheoretische Zugänge in der Diskussion, Hrsg. Clemens Kroneberg, Thomas Schwinn und Jens Greve, 421-432. Wiesbaden: VS Verlag für Sozialwissenschaften.

Schwinn, Thomas, Clemens Kroneberg und Jens Greve. Hrsg. 2011. Soziale Differenzierung. Handlungstheoretische Zugänge in der Diskussion. Wiesbaden: VS Verlag für Sozialwissenschaften.

Simon, Herbert. 1962. The architecture of complexity. American Philosophical Society 106:468-482. 
Smith, Greg. 2012. Why I left Goldman Sachs or: How the world's most powerful bank made a killing but lost its Soul. New York: Central Publications.

Streeck, Wolfgang. 2011. The crises of democratic capitalism. New Left Review 71:5-29.

Streeck, Wolfgang. 2013. Die Krise der Staatsfinanzen: Demokratieversagen? Kapitalismusversagen. Dms - der moderne Staat 6:7-20.

Teubner, Gunther, und Helmut Willke. 1984. Kontext und Autonomie. Gesellschaftliche Selbststeuerung durch reflexives Recht. Zeitschrift für Rechtssoziologie 5:4-35.

Turner, Adair, Andrew Haldane, Paul Woolley, Sushil Wadhwani, Charles Goodhart, Andrew Smithers und Richard Layard. 2010. The future of finance: The LSE report. London: London School of Economics and Political Science.

Willke, Helmut. 2011. Transparency after the financial crisis. In Transparenz. Multidisziplinäre Durchsichten durch Phänomene und Theorien des Undurchsichtigen, Hrsg Eckhard Schröter, Stephan Jansen und Nico Stehr, 56-81. Wiesbaden: VS Verlag für Sozialwissenschaften.

Windolf, Paul. Hrsg. 2005. Finanzmarkt-Kapitalismus. Analysen zum Wandel von Produktionsregimes. Kölner Zeitschrift für Soziologie und Sozialpsychologie, Sonderheft 45. Wiesbaden: VS Verlag für Sozialwissenschaften.

Renate Mayntz, 1929, Dr. phil. habil., Dr. h.c. mult, Honorarprofessor Universität zu Köln und Direktor emeritus des Max-Planck-Instituts für Gesellschaftsforschung. Fachgebiet: Sozialwissenschaft (Venia in Soziologie). Letzte Veröffentlichungen: Crisis and Control - Institutional Change in Financial Market Regulation (Hrsg.). Frankfurt 2012; Über Governance. Institutionen und Prozesse politischer Regelung. Frankfurt 2009; Sozialwissenschaftliches Erklären. Probleme der Theoriebildung und Methodologie. Frankfurt 2009. 